\title{
Modeling the Frequency Dependence of the Effective Path Length Adjustment Factor for Prediction of Rain Attenuation on Earth-Space Paths
}

\author{
G. A. Brost \\ Air Force Research Laboratory \\ 525 Brooks Rd, Rome NY 13441 USA \\ george.brost@rl.af.mil
}

\begin{abstract}
A physical structure model is utilized to analyze the frequency dependence of the adjustment factor associated with empirical attenuation models for earth-space paths. The frequency dependence seen in empirical models comes in part from the frequency dependent specific attenuation. Melting layer and cloud attenuation that is inherent in the data give rise to significant frequency dependence at higher exceedance probabilities.
\end{abstract}

\section{Keywords-attenuation; radio propagation.}

\section{INTRODUCTION}

Empirical models relate measured attenuation statistics to the measured point rainfall rate statistics:

$$
A_{p}=k R_{p}^{\alpha} \cdot L_{S} \cdot A F
$$

where $\mathrm{k}$ and $\alpha$ are the frequency dependent parameters for specific rain attenuation and $L_{S}$ is the slant path length through the rain, and $A F$ is the adjustment factor whose parameters are determined by regression analysis. Various functional forms and terminologies have been applied to the adjustment factor. It may be interpreted as an effective path length, an effective rain rate, or a combination of both. Depending on assumptions and choice of the functional form, $A F$ may[1,2] or may not[3] express a frequency dependence.

Kheirallah et al.[4] noted the existence of a frequency dependence of the effective path length in some data. They analyzed this issue to find an expression for the frequency dependence. However, their analysis had several questionable assumptions including the assumption that the specific attenuation parameter $\alpha$ is approximately independent of frequency.

In this paper we examine the frequency dependence of the effective path length by the use of a physical rain model. Since other sources of attenuation are present in the empirical data, we also include the melting layer and cloud attenuation in the analysis. It will be shown that all of these sources of attenuation provide a physical basis for the frequency dependence, consistent with that found in the empirical models.

\section{ANALYSIS}

A. Model

The probability that the attenuation due to rain along the earth space path is greater than $\mathrm{A}^{\prime}$ can be expressed as

$$
P_{A}\left(A^{\prime}\right)=\int_{0}^{\infty} \int_{-\infty}^{\infty} \int_{-\infty}^{\infty} P_{S}\left(R_{m}\right) S_{A} d x d y d R_{m}
$$

Here $P_{S}\left(R_{m}\right)$ is the probability per unit area, that a rain cell with peak rainfall rate $R_{m}$ will occur. $P_{S}\left(R_{m}\right)$ has dimensions of $\mathrm{km}^{-2}(\mathrm{~mm} / \mathrm{hr})^{-1}$. $\quad S_{A}\left(x, y, A^{\prime}, R_{M}\right)$ is the dimensionless function that indicates if a rain cell with peak rain rate $R_{m}$ located at the point $(x, y)$ will result in an attenuation $A>A^{\prime}$. Its spatial integration represents the area over which a rain cell with peak rain rate $R_{m}$ will result in an attenuation $A>A^{\prime}$. The expressions for $P_{S}$ and $S_{A}$ depend upon the rain cell model (ie. shape and size). $P_{S}$ also depends upon the rain rate statistics. In reference [5] horizontal structures of exponential, Gaussian, and uniform cylinder were compared and it was shown that the horizontal shape has little impact on the long term statistics. Consequently we use a uniform cylinder defined by

$$
R(r)=R_{m}, r \leq \lambda
$$

where $R_{m}$ is the peak rain rate, $r$ is the horizontal distance from the rain cell center. Let $P_{R}\left(R^{\prime}\right)$ be the probability distribution for rain rate where $P_{R}\left(R^{\prime}\right)$ is the probability that $R>$ $R^{\prime} . P_{R}\left(R^{\prime}\right)$ can be expressed as

$$
\mathrm{P}_{\mathrm{R}}\left(R^{\prime}\right)=\int_{R^{\prime}}^{\infty} P_{S}\left(R_{m}\right) S_{R}\left(R^{\prime}, R_{m}\right) d R_{m}
$$

where $S_{R}\left(R^{\prime}, R_{m}\right)$ is the area for which $R>R^{\prime}$, given a peak rain rate of $R_{m}$. Equation (4) relates the attenuation statistics to the rain rate statistics through the dependence of $P_{S}$ on $P_{R}$, and assures that the local rain rate distribution is maintained. For the uniform rain cell $P_{S}$ is simply related to the probability density of the rain rate

$$
P_{S}\left(R_{m}\right)=\frac{P\left(R_{m}\right)}{\pi\left[\lambda\left(R_{m}\right)\right]^{2}}
$$

where

$$
P\left(R_{m}\right)=\left.\frac{-d}{d R^{\prime}} P_{R}\left(R^{\prime}\right)\right|_{R^{\prime}=R_{m}}
$$


We use the analytical rain rate distribution function used in the ITU-R P.837 model [6] which has the form

$$
P_{R}(R)=P_{0} e^{-a R \frac{1+b R}{1+c R}}
$$

where the parameters $\left[\mathrm{a}, \mathrm{b}, \mathrm{c}, \mathrm{P}_{0}\right]$ are calculated from the meteorological quantities $\left[\mathrm{M}_{\mathrm{T}}, \beta, \mathrm{P}_{6 \mathrm{hr}}\right]$ derived from the ITU database. The spatial integration in equation (1) is simplified through the use of a kernel for a uniform cylindrical cell model which can be expressed as

$$
\begin{gathered}
S_{U}\left(A^{\prime}, R\right)= \\
\left(\left(L_{G}-1.5 L_{A}\right) Y+\left[0.5 D^{2}(R) \operatorname{atan}\left(\frac{Y}{L_{A}}\right)\right]\right) \Phi\left(L_{G}-L_{A}\right)
\end{gathered}
$$

where $L_{G}$ is the horizontal projection of the slant path length, $D(R)$ is the cell diameter, $L\left(A^{\prime}, R\right)$ is the path length required to reach an attenuation $A^{\prime}$

$$
\begin{aligned}
& L_{A}=L\left(A^{\prime}, R\right)=\frac{A^{\prime} \cos \theta}{\kappa R^{\alpha}}, \\
& \text { and } \\
& \begin{array}{c}
Y=Y\left(A^{\prime}, R\right)=\sqrt{D^{2}(R)-L^{2}(A, R)}, \\
D(R)>L\left(A^{\prime}, R\right)
\end{array} \\
& =0 \text {, otherwise. }
\end{aligned}
$$

To complete the model description we need a function that relates the cell size to the peak rain rate $R_{m}$. We use an equation that relates the radius of a uniform rain cell to the peak rain rate

$$
\lambda\left(R_{M}\right)=14 R_{M}^{-.45}
$$

This approximates the cell model given by Capsoni et al[7] at rain rates above $7 \mathrm{~mm} / \mathrm{hr}$ and is still well behaved at lower rain rates, and is similar to the model of Misme and Walteufel [8]. The melting layer model of Capsoni et al [9] is included for stratiform rain. This approach increases the rain height by a frequency dependent equivalent bright band height

$$
H_{B B}(f)=4.58 e^{-0.0675 f}+0.51(k m)
$$

The threshold $\mathrm{R}_{\mathrm{T}}$ that separates stratiform and convective rain is determined by rain rate that is consistent with $\beta$ given by the ITU rain rate parameters. When correlated cloud attenuation is also included the cloud attenuation is combined on an equal probability basis with the rain attenuation.

$$
A_{R C}=A_{R}+.5 A_{C}
$$

Here the liquid cloud attenuation is one half the attenuation as given by the ITU model.[9] The excess attenuation then is calculated as

$$
A_{E}=\sqrt{A_{R C}^{2}+.25 A_{C}^{2}} .
$$

Equation (2) can be inverted to solve for $A_{p}$ and inserted into equation (1) to solve for $\mathrm{AF}$

\section{B. Results}

In the following analysis we calculate the attenuation and adjustment factor for conditions at Spino d'Adda and elevation angle of $37.7^{\circ}$. The frequency dependence of the adjustment factor for Brazil-08[2] and China-10[1] empirical models is shown in Figure 1. The Brazil-08 model exhibits a frequency dependence in the adjustment factor through its dependence on the rain specific attenuation parameter. The adjustment factor in the China-10 model has an explicit dependence on frequency.
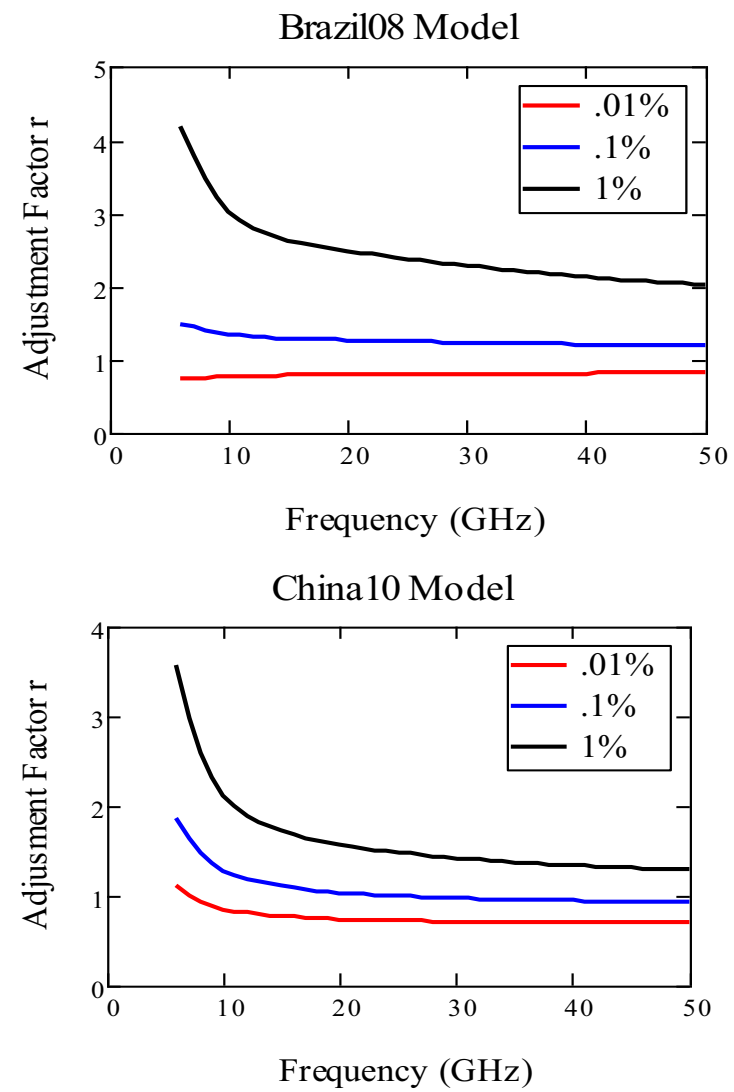

Figure 1. Frequency dependence of the adjustment factor for the China-10 and the Brazil-08 rain attenuation prediction models.

In Figure 2 we show the the frequency dependence calculated for the case of rain only attenuation for different exceedance probabilities. These plots exhibit the general trend shown by the empirical models of mild frequency dependence except at the lower frequencies, but the magnitude is less compared to the China- 10 model for the $\mathrm{p}=0.01 \%$ and $\mathrm{p}=0.1 \%$ cases, and much less than the China-10 and Brazil-08 models for the $\mathrm{p}=1 \%$ case. Insight regarding the source of this frequency dependence is gained by examining the distribution of rain- 
rates that contribute to the attenuation $A_{p}$. This is shown in Figure 3. While the form of equation (1) uses only the rain rate $R_{p}$, there is actually a significant distribution of rain rates that contribute to $A_{p}$. This is coupled with the power law dependence of specific attenuation which is frequency dependent. The dependence of the adjustment factor on $\alpha$ is further revealed by setting $\alpha$ to a constant value $\alpha=1$. In this case there is no frequency dependence in the adjustment factor.

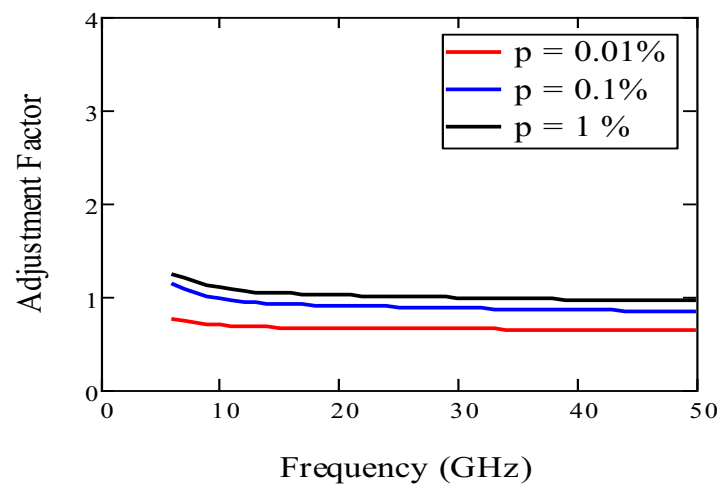

Figure 2. Frequency dependence of adjustment factor calculated for rain only rain cell model.

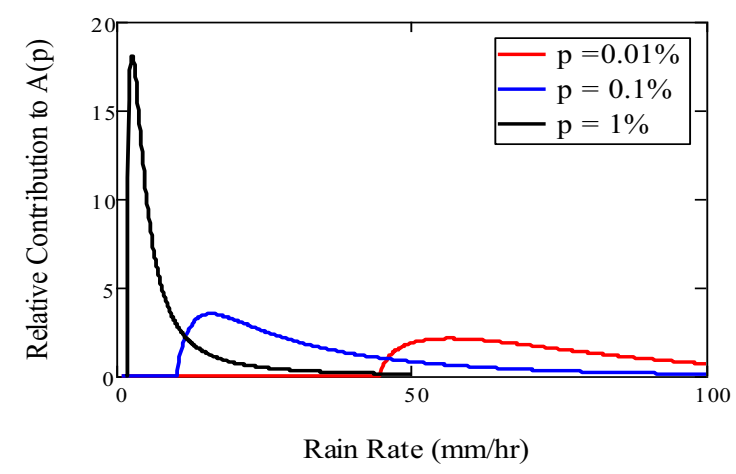

Figure 3. Relative contribution of rain rates to the attenuation $A_{p}$

We next consider the fact that the attenuation data used for the regression analysis includes attenuation due to the melting layer and clouds. Thus the empirical models are really excess attenuation models, not simply rain only attenuation prediction models. We examine the impact on the frequency dependence of the adjustment factor. In Figures 4 and 5 we show calculated adjustment factor in which the melting layer and cloud attenuation are included. Inclusion of the melting layer increases the magnitude of the frequency dependence at the higher percentages but has no affect for $\mathrm{p}=0.01 \%$ due to the high rain rates associated with this attenuation level. The relative contribution of clouds to the total attenuation increases with $\mathrm{p}$, and has more impact on the adjustment factor at the higher exceedance probabilities.

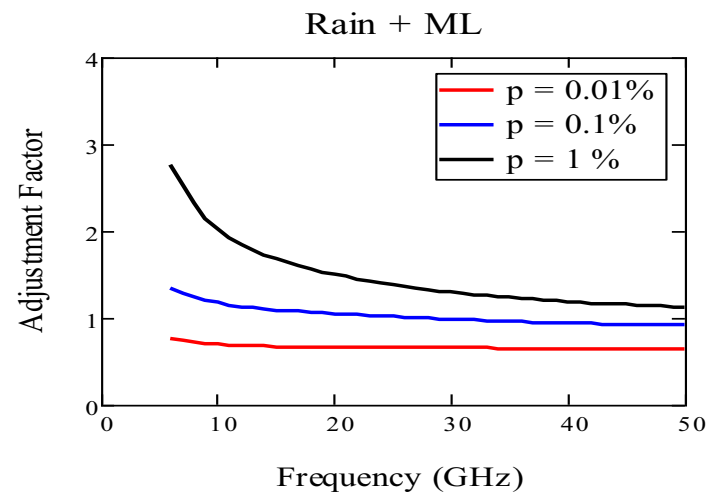

Figure 4. Calculated adjustment factor when the melting layer is included.

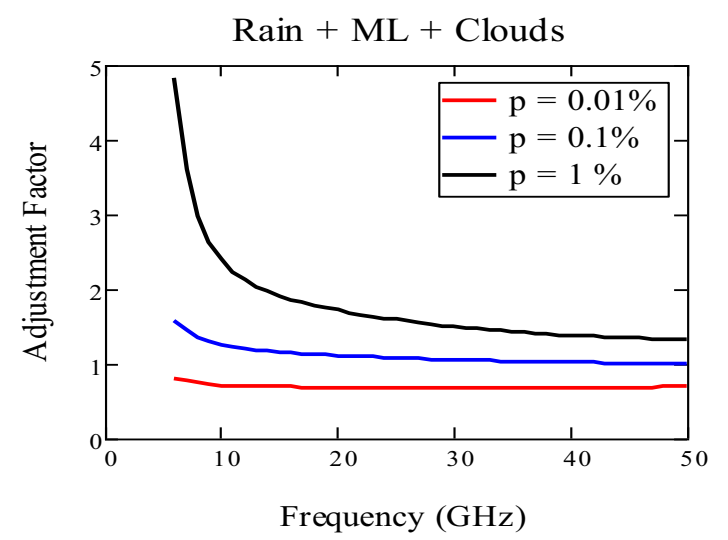

Figure 5. Calculated adjustment factor when the melting layer and cloud attenuation are included.

While the total attenuation due to the melting layer and clouds increases with frequency, the frequency dependence of the adjustment factor reflects the attenuation relative to the rain attenuation. This is shown for the melting layer in Figs. 6 and 7 and for clouds in Fig. 8. Figure 6 shows the frequency dependence of the equivalent bright band height that is added to the rain height in the melting layer model of Capsoni et al.[9] that is used here. Figure 7 shows the specific attenuation of the melting layer relative to the specific attenuation of rain as given by the melting layer model of Zahng et al. [10] for rain rates at $\mathrm{p}=0.01 \%, 0.1 \%$ and $1 \%(43.4,11.2$ and 2.1 $\mathrm{mm} / \mathrm{hr}$ ). Figure 8 shows the specific attenuation of cloud attenuation relative to the specific attenuation of rain for rainfall rates at different percentages.

\section{SUMMARY AND CONCLUSIONS}

We have applied physical models to analyze the expected frequency dependence of the adjustment factor associated with empirical attenuation models. The calculated frequency dependence is in general agreement with empirical models that allow for the possibility of a frequency dependence. The analysis showed the physical basis for the behavior, and that 
there are multiple factors. It is due in part to the artificial mapping of attenuation at percentage $p$ to the corresponding rainfall rate at $p$, and treating all attenuation as if it is due to that single rainfall rate. A distribution of rainfall rates contribute to the attenuation. This, coupled with a frequency dependent power law relation for rain specific attenuation, gives rise to a dependence that follows that of $\alpha$. A more significant contribution comes from sources of attenuation other than rain. The relative contributions from the melting layer and clouds decrease with frequency and increase with exceedance probability. As these effects are present in the measured attenuation data they are also inherent in the empirical "rain" attenuation models. Empirical models should therefore allow for frequency dependence in the adjustment factor.

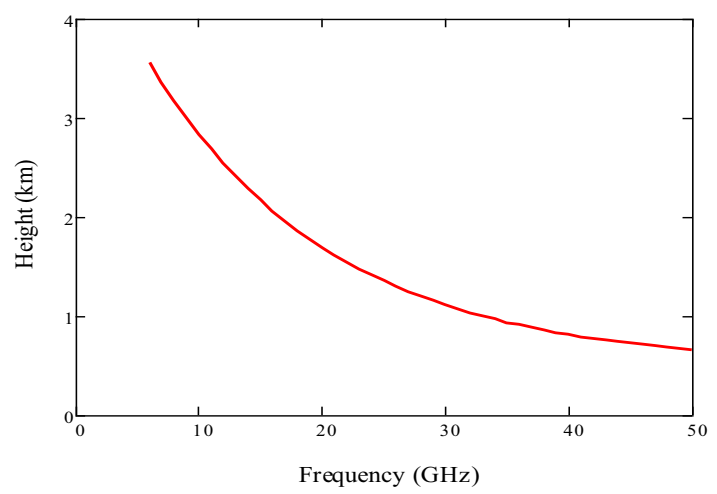

Figure 6. Frequency dependence of melting layer attenuation. Equivalent bright is from band height from Capsoni et al. [9].

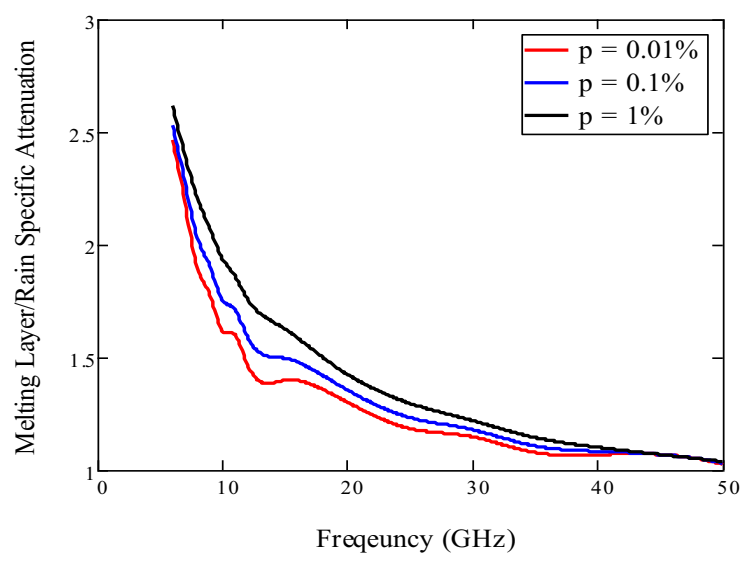

Figure 7. Frequency dependence of melting layer attenuation. Ratio of melting layer and rain specific attenuations for rain rates at different percentages from Zahng et al. [10].

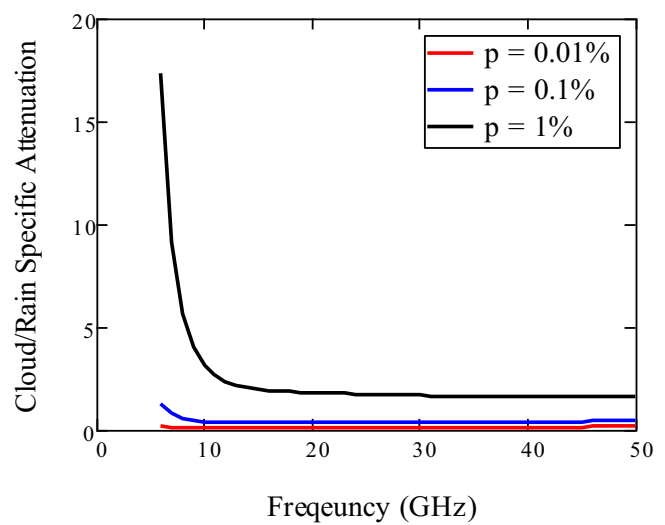

Figure 8 Ratio of cloud specific attenuation to rain specific attenuation at rainfall rates at different percentages.

\section{REFERENCES}

[1] International Telecommunication Union, ITU-R Document 3M/145(2010), Geneva Switzerland [2] International Telecommunication Union, ITU-R Document

[3] International Telecommunication Union, ITU-R Document 3M/40 (2003), Geneva Switzerland

[4] H. Kheirallah J. Knight, R. Olsen, K. McCormic, and B. Segal, "Frequency dependence of effective path length in prediction of rain attenuation statistics", Electron. Lett., vol. 16, pp. 448-450, 1980

[5] G. Brost, "Influence of the Rain Cell Structure on Attenuation Predictions on Earth-Space Paths “, European Conference on Antennas and Propagation, Prague, March 2012.

[6] Blarzino, G.; Castanet, L.; Luini, L.; Capsoni, C.; Martellucci, A.; "Development of a new global rainfall rate model based on ERA40, TRMM, GPCC and GPCP products," Antennas and Propagation, 2009. EuCAP 2009. 3rd European Conference on , vol., no., pp.671-675, 2327 March 2009.

[7] C. Capsoni, F. Fedi, C. Magistroni, A. Pawlina, and A. Paraboni, "Data and theory for a new model of the horizontal structure of rain cells for propagation applications", Radio Science, vol 22, (3) pp 395-404, 1987.

[8] P. Misme and P. Waldteufel, "A model for attenuation by precipitation on microwave earth-space link", Radio Science, vol 15 pp. 655-665, 1980.

[9] C. Capsoni, L. Luini, A. Paraboni, C. Riva, and A. Martellucci, “ A new prediction model of rain attenuation that separately accounts for stratiform and convective rain", IEEE Tans. Ant. Prop., vol 57, pp. 196204, 2009.

[10] International Telecommunication Union-Radiocommunication (ITU-R) (2009), Attenuation due to clouds and fog., Recomm ITU-R P.840-4, Geneva, Switzerland.

[11] W. Zhang, S. Karhu, and E. Salonen, "Predictions of radiowave attenuations due to a melting layer of precipitation", IEEE Trans. Ant. Prop., vol 42, pp. 492-500, 1994.

[12] A. Dissanayake, J. Allnutt, and F. Haidara, "A prediction model that combines rain attenuation and other propagation impairments along earth-satellite paths", IEEE Trans. Ant. Prop., vol. 45, pp. 1546-1558, 1997. 